\title{
Sicherheit von HPV-Impfungen: eine Erklärung der FIGO
} Juli 2013

\section{Safety of HPV vaccination: A FIGO Statement}

July, 2013

Autoren

Institute

\author{
S. Arulkumaran ${ }^{1}$, L. Denny ${ }^{2}$, J. Cain ${ }^{3}$ \\ 1 Präsident FIGO \\ ${ }^{2}$ Vorsitzende, FIGO Komitee für Gynäkologische Onkologie \\ ${ }^{3}$ Vorsitzende, Unterausschuss für Verhütung von Gebärmutterhalskrebs
}

\section{Erstpublikation}

Übersetzung der Erstpublikation in International Journal of Gynecology and Obstetrics 2013; 123: $187-188$
Bibliografie

DOI http://dx.doi.org/

10.1055/s-0033-1351076

Geburtsh Frauenheilk 2014; 74 :

230-232 @ Georg Thieme

Verlag KG Stuttgart · New York . ISSN 0016-5751

Korrespondenzadresse

Prof. Dr. med.

Matthias W. Beckmann

Frauenklinik

Universitätsklinikum Erlangen

Universitätsstraße 21-23

91054 Erlangen

fk-direktion@uk-erlangen.de
Impfungen gegen humane Papillomaviren werden in vielen Ländern eingesetzt; weltweit wurden bereits mehr als 175 Millionen Dosen verabreicht. Es gibt ausführliche Daten über die Sicherheit dieser Impfungen vor und nach ihrer Zulassung. Folgende Informationsquellen wurden für die Sicherheitsbewertung von HPV-Impfstoffen herangezogen:

1. randomisierte kontrollierte klinische Studien

2. Das US-amerikanische Meldesystem VAERS

(Vaccine Adverse Event Reporting System), das unerwünschte Ereignisse registriert, die im Zusammenhang mit Impfungen auftreten.

3. Das Australische Meldesystem SAEFVIC (Surveillance of Adverse Events Following Vaccination in the Community), Victoria, Australien, welches 2007 ins Leben gerufen wurde.

4. Die Europäische Arzneimittelagentur (EMA), 2010

5. Daten zu den Erfahrungen mit Impfstoffen nach ihrer Zulassung aus

a) passiver Überwachung

b) passiver und aktiver Überwachung c) bevölkerungsbezogener epidemiologischer Überwachung

- Randomisierte kontrollierte Studien, die vor der Zulassung in einigen Ländern durchgeführt wurden und die inzwischen durch neue Studienergebnisse aus den Ländern Korea, China, Japan und Vietnam ergänzt wurden

- Zu den Sicherheits-Endpunkten zählten lokale und systemische unerwünschte Ereignisse (UE), schwerwiegende UE, Todesfälle, neu auftretende Beschwerden, einschließlich chronischer und/oder Autoimmunerkrankungen sowie Angaben zum Schwangerschaftsverlauf.

- Ergebnisse von klinischen Studien zur Sicherheit einer Vierfach-Impfung:

- Metaanalysen von Studien mit mehr als 20000 Frauen im Alter von 9-26 Jahren und 1350 Männern im Alter von 9-16 Jahren aus Europa, Nord- und Südamerika zeigten, dass eine Reaktion an der Injektionsstelle wie z. B. Schmerzen, Erythem und Schwellungen häufiger bei Personen auftraten, die einen Impf- 
stoff erhalten hatten, verglichen mit Personen, die adjuvante oder Placeboimpfungen erhielten. In fast allen Fällen waren die Symptome selbstlimitierend und innerhalb von 48 Stunden abgeklungen.

- Beim Auftreten allgemeiner unerwünschter Ereignisse wie Kopfschmerzen, Fieber und Übelkeit gab es keinen Unterschied zwischen den Gruppen.

- Es gab ebenfalls keinen Unterschied hinsichtlich der Häufigkeit des Auftretens von SUE im Allgemeinen bzw. in verschiedenen Organsystemen über einen mittleren Beobachtungszeitraum von knapp 4 Jahren.

- Sowohl in der Impf- als auch in der Placebogruppe betrug die Mortalitätsrate $0,1 \%$; kein Todesfall hing ursächlich mit der Impfung zusammen.

- Insgesamt unterschied sich der Prozentsatz der Teilnehmer, die über neu auftretende Autoimmunreaktionen berichteten, nicht zwischen beiden Gruppen (2,4\% in beiden Gruppen) über einen Beobachtungszeitraum von 4 Jahren.

- Ergebnisse von klinischen Studien zur Sicherheit der bivalenten Impfung

- Das Sicherheitsprofil von bivalenten Impfstoffen wurde in klinischen Studien untersucht und entspricht dem Sicherheitsprofil der Vierfach-Impfung; die am häufigsten genannten Beschwerden waren Schmerzen an der Injektionsstelle (aufgetreten in bis zu 97\% aller geimpften Personen; die Daten wurden aus 11 Studien mit mehr als 30000 Frauen älter als 10 Jahre gepoolt), gefolgt von Erythemen und Schwellungen.

- Es gab keine signifikanten Unterschiede zwischen der geimpften Gruppe und der Kontrollgruppe bezüglich der Häufigkeit des Auftretens von SUE, unerwünschten Symptomen oder signifikanten Beschwerden.

- Es wurden 5 Todesfälle gemeldet (davon hatte nur eine Person zuvor eine Impfung bekommen); es konnte kein Kausalzusammenhang zwischen den Todesfällen und der Impfung festgestellt werden.

- In der Nachbeobachtung über einen Zeitraum von 4 Jahren gab es keinen Unterschied zwischen beiden Gruppen hinsichtlich des Auftretens von SUE, von neu hinzugekommenen chronischen Erkrankungen oder von neu hinzugekommenen Autoimmunerkrankungen.

- Zusammenfassung:

- Alle randomisierten klinischen Studien sowohl von bivalenten als auch von tetravalenten Impfstoffen weisen ein hervorragendes Sicherheitsprofil auf. Die häufigsten Beschwerden waren Schmerzen an der Injektionsstelle, welche größtenteils selbstlimitierend waren und spontan abheilten.

\section{Besondere Umstände}

$\nabla$

\section{- Sicherheit während der Schwangerschaft}

- Die Verabreichung einer HPV-Impfung wird bei schwangeren Frauen generell nicht empfohlen; nichtsdestoweniger wurden einige Teilnehmerinnen während der klinischen Studie schwanger.

- Es wurden Schwangerschaftsregister für beide handelsüblichen Impfstoffe eingerichtet.

- Die Schwangerschaftsverläufe von Frauen in den Gruppen „bivalente Impfung“ und „tetravalente Impfung“ unterschieden sich nicht von Frauen in der Kontrollgruppe und der allgemeinen Bevölkerung.
- Es gibt keinerlei Hinweise, weder in klinischen Studien noch in den nach der Zulassung gesammelten Daten, dass der Anteil angeborener Fehlbildungen oder der Prozentsatz geburtshilflicher Komplikationen bei geimpften Frauen höher war.

- Daten nach der Zulassung

- Daten aus passiven, aktiven und bevölkerungsbezogenen epidemiologischen Überwachungsstudien, die seit 2006 gesammelt wurden, zeigten keinerlei Unterschiede in der Häufigkeit des Auftretens von Leiden wie das Guillain-BarréSyndrom, Schlaganfall, Blinddarmentzündungen, Anfälle, allergischen Reaktionen, anaphylaktischen Reaktionen und venösen Thromboembolien - die Daten wurden der Vaccine Safety Datalink-Kohortenstudie entnommen, die insgesamt 600558 Dosen des tetravalenten Impfstoffs verzeichnet. Eine Durchsicht der Krankenakten aller 8 geimpften Fälle in der Altersgruppe 9-17 Jahre, die potenziell eine venöse Thromboembolie (VTE) aufwiesen, zeigte, dass in 5 Fällen VTE gemäß Standarddefinition vorlag; alle 5 Personen wiesen bekannte Risikofaktoren für eine VTE auf (Einnahme von oralen Kontrazeptiva, Koagulationsstörungen, Rauchen, Adipositas oder längerer Krankenhausaufenthalt).

\section{Besondere Nebenwirkungen}

$\nabla$

\section{- Synkope}

- Beobachtungsstudien nach der Zulassung in den Niederlanden, Australien und Italien berichteten über eine überproportional höhere Rate von Fällen mit Synkope nach einer HPV-Impfung verglichen mit randomisierten kontrollierten Studien vor der Zulassung.

- Nach einer HPV-Impfung wird häufig von Schmerzen an der Injektionsstelle berichtet; eine Synkope gilt eher als ungewöhnliche Nebenwirkung und nicht als unerwünschtes Ereignis nach einer Impfung; es wird empfohlen, Vorsichtsmaßnahmen zu treffen (z.B. Hinsetzen nach der Impfung), um Stürzen wegen Schwindelgefühls oder kurzer Ohnmacht vorzubeugen. Dies betont die Notwendigkeit einer engmaschigen Überwachung während 15-30 Minuten nach einer Impfung.

- Synkopen scheinen nicht häufiger in HPV-geimpften Frauen vorzukommen als in anderen Gruppen nach einer Impfung mit anderen Impfstoffen. Die US-amerikanischen Centers for Disease Control and Prevention haben nach der Zulassung die Häufigkeit des Auftretens einer Synkope bei 3 Impfstoffen ausgewertet - dem tetravalenten HPV-Impfstoff, bei der Verabreichung von Einzeldosen des konjugierten Meningokokken-Vierfach-Impfstoffs und bei der Verabreichung von Einzeldosen des Diphtherie-, Tetanus- und PertussisKombinations-Impfstoffs - und fanden, dass Synkope ähnlich häufig bei allen 3 Impfstoffen auftrat. Sie empfahlen, alle Patienten für mindestens 15 Minuten nach Verabreichung der Impfung zu beobachten (eine Empfehlung, die auch vom Advisory Committee on Immunization Practices getragen wird), um synkopebedingten Verletzungen vorzubeugen.

\section{- Anaphylaxie}

- Obwohl sehr selten, kann Anaphylaxie nach jeder Impfung auftreten, hervorgerufen entweder durch ein Antigen und/ oder ein Impfstoff-Adjuvans.

- In nationalen passiven Beobachtungs- und populationsbezogenen Studien trat Anaphylaxie nach HPV-Impfung gleich- 
bleibend häufig auf; die Häufigkeit bewegte sich in einer geschätzten Bandbreite von 1-10 Fälle pro Millionen Dosen, eine Zahl, die gut vergleichbar war mit den Ergebnissen von anderen Impfstoffen wie beispielsweise Tetanus-, Diphtherie- und Pertussis-Kombinations-Impfstoffen.

- Guillain-Barré-Syndrom (GBS)

- Eine Überprüfung von GBS-Fällen, die dem US-amerikanischen VAERS berichtet wurden, deutete auf eine möglicherweise 2,5-10-mal höhere Rate von GBS-Fällen innerhalb von 6 Wochen nach Impfung mit dem Vierfach-Impfstoff hin, verglichen mit der erwarteten Rate für die allgemeine Bevölkerung. Nach umfangreicher Untersuchungen kam man schließlich zum Schluss, dass wegen des Fehlens von Kontrollgruppen weitere Studien nötig wären, auch um die zeitliche Verbindung mit der Impfung zu prüfen. Die anschließend durchgeführten bevölkerungsbezogenen Studien mit umfangreichen Untersuchungen von Einzelfällen haben keine Hinweise erbracht, dass die Rate signifikant höher ist als in einer allgemeinen Bevölkerung mit heranwachsenden und jungen Frauen zu erwarten war.

- Venöse Thromboembolie (VTE)

- Vom Auftreten einer VTE nach der Impfung wurde nur sehr selten berichtet; bei allen Einzelfällen spielten zusätzliche Risikofaktoren für VTE eine Rolle, beispielsweise die Einnahme oraler Kontrazeptiva. Hinzu kam noch, dass die zeitliche Verbindung zur Impfstoffverabreichung stark variierte; damit ist es sehr unwahrscheinlich, dass es sich hier um eine mit diesem Impfstoff verbundene Komplikation handelt.

- Komplexes regionales Schmerzsyndrom (complex regional pain syndrome - CRPS)

- Zu den häufig genannten UE, die nach einer Immunisierung auftreten, gehören lokale Schmerzen an der Injektionsstelle; die Entstehung eines CPRS wurde bislang nur bei Kindern beschrieben, die zuvor eine Immunisierung mit Röteln oder Hepatitis-B-Impfstoffen erhalten hatten. CPRS ist ein klinisches Syndrom, das eine oder mehrere Extremitäten in Mitleidenschaft ziehen kann und durch anhaltende Schmerzen gekennzeichnet ist, die in keinem Verhältnis zum verursachenden Ereignis stehen, welches oft nur aus einer kleinen Verletzung bestand.

- Bislang wurden dem Surveillance of Adverse Events following Vaccination in the Community 4 Fälle berichtet, nach der Gründung dieser Institution im Jahre 2007 in Victoria, Australien, sowie ein Fall aus dem Vereinigten Königreich.

- Vier dieser 5 Fälle erfüllten die Kriterien für ein CPRS; die Symptome verschwanden bei allen betroffenen Personen nach einem längeren Zeitraum zwischen 5 Tagen und 7 Monaten, ohne dass die Symptome später wiederauftraten oder andere Auswirkungen folgten.
- Es ist wichtig, dieses Syndrom zu kennen, das durch irgendeine Verletzung an den Extremitäten - auch durch eine intramuskuläre Injektion - ausgelöst werden kann.

- Fälle mit chronischen Schmerzen in den Extremitäten wurden auch aus Japan berichtet, wo inzwischen mehr als 8 Millionen Dosen des HPV-Impfstoffs ausgegeben wurden - dieser Frage wird zurzeit intensiv nachgegangen, aber die bisher eingegangenen Daten haben keinen überzeugenden Nachweis erbracht für eine ursächliche Verbindung mit der HPV-Impfung verglichen mit anderen Impfungen.

\section{Fazit}

$\nabla$

- Das globale Beratungskomitee zur Sicherheit von Impfstoffen (Global Advisory Committee on Vaccine Safety [GACVS]) der Weltgesundheitsorganisation (WHO) hat die Sicherheit der HPV-Impfung nochmals am 13. Juni 2013 überprüft, nachdem die letzte Prüfung durch dieses Beratungskomitee im Jahre 2009 stattfand.

- Das Beratungskomitee hat alle verfügbaren Daten zur HPVImpfung sorgfältig geprüft und kam zum Schluss, dass beide handelsüblichen Impfstoffe sicher sind.

- Nach der Prüfung alle verfügbaren Daten unterstützen das FIGO Komitee für Gynäkologische Onkologie und der FIGO Unterausschuss für Verhütung von Gebärmutterhalskrebs die weitere Verabreichung von HPV-Impfstoffen in bestimmten Bevölkerungen.

- Diese Empfehlung wurde vom FIGO-Vorstand genehmigt.

\section{Literatur}

1 http://www.who.int/vaccine_safety/committee/topics/hpv/ 130619HPV_VaccineGACVSstatement.pdf

2 Macartney KK, Chiu C, Georgousakis M et al. Safety of human papillomavirus vaccines: a review. Drug Saf 2013; 36: 393-412

3 Gee J, Naleway A, Shui I et al. Monitoring the safety of quadrivalent human papillomavirus vaccine: findings from the vaccine safety datalink. Vaccine 2011; 29: 8279-8284

4 Klein NP, Hansen J, Chao C et al. Safety of quadrivalent human papillomavirus vaccine administered routinely to females. Arch Pediatr Adolesc Med 2012; 166: 1140-1148

5 Centers for Disease Control and Prevention (CDC). Syncope after vaccination-Unites States, January 2005-July 2007. MMWR Morb Mortal Wkly Rep 2008; 57: 457-460

6 Chang S, O'Connor PM, Slade BA et al. U.S. Postlicensure safety surveillance for adolescent and adult tetanus, diphtheria and acellular pertussis vaccines: $2005-2007$. Vaccine 2013; 31: 1447-1452

7 Richards S, Chailkiadis G, Laksham R et al. Complex regional pain syndrome following immunisation. Arch Dis Child 2012; 97: 913-915 